\title{
Hallazgos anatomopatológicos definitivos en pacientes tiroidectomizados con diagnóstico preoperatorio de neoplasia folicular*
}

\author{
Drs. IGNACIO GOÑI E. ${ }^{1}$, CLAUDIO KRSTULOVIC R. ${ }^{1}$, AUGUSTO LEÓN R. ${ }^{1}$, HERNÁN GONZÁLEZ D. ${ }^{1}$, \\ ANTONIETA SOLAR ${ }^{2}$, CLAUDIA CAMPUSANO M. ${ }^{3}$, EU. CATALINA RUIZ A. ${ }^{1}$ \\ Departamento de Cirugía Oncológica y Maxilofacial, División de Cirugía. \\ Departamento de Anatomía Patológica. \\ 3 Departamento de Endocrinología. \\ Pontificia Universidad Católica de Chile, Santiago, Chile.
}

\begin{abstract}
Definitive anatomopathological findings in thyroidectomized patients with preoperative diagnosis of follicular neoplasm

Background: The finding of follicular neoplasm, using a FNAP, is an indication for partial or total thyroidectomy, to obtain the definitive malignant or benign histology. Frequently, it is possible to identify significant additional histological diagnosis. Aim: To obtain the definitive histological findings in patients with follicular neoplasm by FNAP. Patients and Method: Transversal analysis of 133 patients that underwent to total thyroidectomy between 2003 and 2009, that filled de requirements for adequate histological assessment. Results: In $33.1 \%$ of the treated patients the final diagnosis was indeed a follicular neoplasm (adenoma in $26.3 \%$ and cancer in $6.8 \%$ ). In the $51.9 \%$ the finding was follicular colloidal hyperplasia and other thyroid cancer in $8.3 \%$. The total malignant prevalence in the whole gland was $29.3 \%$. Conclusions: The thyroidectomy is the treatment of choice and the final diagnostic procedure for these patients. The histological findings of cancer different from follicular not only in the punctioned nodule are a secondary and an additional argument for reinforcing the surgical indication.
\end{abstract}

Key words: Thyroidectomy, thyroid cancer, thyroid follicular neoplasm.

\section{Resumen}

Introducción: El hallazgo de una neoplasia folicular por PAAF, obliga a realizar una tiroidectomía parcial o total, para definir la naturaleza maligna o benigna definitiva de la lesión tiroidea. Junto a este diagnóstico preoperatorio se identifican finalmente con alta frecuencia lesiones histológicas adicionales. Objetivo: Conocer y describir los hallazgos anatomopatológicos definitivos que se encuentran en tiroidectomías por neoplasias foliculares diagnosticadas por PAAF. Materiales y Métodos: Revisión transversal de las biopsias definitivas de 133 pacientes sometidos a tiroidectomía total entre 2003 y 2009, que cumplieron los requisitos

\footnotetext{
* Recibido el 6 de octubre de 2011 y aceptado para publicación el 7 de noviembre de 2011.

Correspondencia: Dr. Ignacio Goñi E.

Marcoleta 367, Santiago, Chile. Fax: (2) 639-6395

igoni@med.puc.cl
} 
establecidos para evaluar la histología definitiva del nódulo puncionado y de la glándula tiroides completa. Resultados: En el 33,1\% de los pacientes el diagnóstico definitivo del nódulo puncionado fue efectivamente una neoplasia folicular (adenoma en el 26,3\% y cáncer en el 6,8\%). El 51,9\% correspondió a hiperplasia folicular y el 8,3\% otro cáncer. La prevalencia de malignidad final en la glándula completa fue de un $29,3 \%$. Conclusiones: Siendo la indicación de tiroidectomía en estos pacientes un tratamiento y procedimiento diagnóstico aceptado y necesario, se concluye que la alta prevalencia de lesiones malignas $(29,3 \%)$ tanto en el nódulo puncionado como, adicionalmente, en el resto de la glándula, reforzaría la necesidad de este tratamiento quirúrgico.

Palabras clave: Tiroidectomía, cáncer de tiroides, neoplasia folicular.

\section{Introducción}

Los tumores tiroideos, benignos y malignos, corresponden a las neoplasias endocrinas más frecuentes ${ }^{1}$. Dentro de éstos, destaca el diagnóstico de "neoplasia folicular" en relación a un nódulo tiroideo y que se refiere a sus características histopatológicas obtenidas por punción aspirativa con agua fina (PAAF), que hacen plantear la posibilidad de un adenoma o cáncer folicular. Este hallazgo ha sido definido como un resultado diagnóstico "indeterminado", junto a la presencia de células atípicas y de Hürthle y a la sospecha de cáncer papilar². La Asociación Americana de Tiroides y el Instituto Nacional del Cáncer (NCI, EEUU), agregan a esta categoría de resultado de la PAAF, los informes "no diagnósticos", "malignos" con sospecha mayor de $95 \%$, "benigna" y algunas modificaciones adicionales ${ }^{3}$. Actualmente, se considera que la diferenciación entre adenoma y cáncer folicular de tiroides sólo es posible de realizarla con el estudio completo del nódulo puncionado, requiriéndose de una tiroidectomía parcial o total, que permita un estudio histológico definitivo ${ }^{4}$. Por otro lado, en la literatura se han estudiado marcadores inmunohistoquímicos y genéticos para la diferenciación preoperatoria de nódulos tiroideos de diversa histología. Los resultados han sido controvertidos y persiste el concepto de que no reemplazan el estudio histopatológico tradicional. Por otro lado, requieren de la utilización de varios marcadores simultáneamente y agregan un costo importante al estudio ${ }^{1,4,5}$. De esta forma, continúa siendo necesaria la cirugía como procedimiento diagnóstico y terapéutico en estos $\operatorname{casos}^{1-8}$. La diferencia histológica fundamental entre el adenoma y el cáncer folicular es la presencia en éste de invasión capsular y vascular ${ }^{4}$. Se ha descrito que en alrededor de un $10 \%$ de las neoplasias foliculares diagnósticas por PAAF la histología definitiva será un cáncer folicular ${ }^{4}$ y el resto, patología benigna o maligna no folicular. Las indicaciones de tiroidectomía en estos pacientes son el riesgo de cáncer en la lesión puncionada y en el resto de la glándula extirpada (hasta en un $30 \%$ en algunas series) ${ }^{2}$. El objetivo de este trabajo es conocer y describir los hallazgos anatomopatológicos definitivos que se encuentran en tiroidectomías por neoplasias foliculares diagnosticadas por PAAF.

\section{Material y Método}

Este estudio fue desarrollado en base a revisión de las biopsias y registros de los pacientes operados en nuestro centro con diagnóstico preoperatorio de "neoplasia folicular" y que cumplieran con los siguientes criterios de inclusión: 1) identificación clara del nódulo puncionado y 2) biopsia definitiva que identificara la histología del nódulo puncionado y de la muestra total. No se consideraron en el estudio marcadores inmunohistoquímicos.

Los casos corresponden a pacientes operados entre los años 2003 y 2009 que cumplieron con los criterios descritos. A todos ellos se les realizó una tiroidectomía total, como conducta estándar.

Los resultados fueron tabulados según la histología definitiva del nódulo puncionado y a los hallazgos en la muestra de tiroides total, y fueron agrupados en patología maligna y no maligna para efecto del análisis.

La significancia estadística fue calculada por test $\chi^{2}$.

El análisis descriptivo de las variables fue realizado con el Statistical Package for Social Sciences version 17.0 (SPSS Inc, Chicago, IL, USA)

\section{Resultados}

Para la evaluación histopatológica definitiva de los pacientes con PAAF informada como "neoplasia folicular", se estudiaron 133 casos, recolectados entre enero de 2003 y septiembre de 2009, con identificación segura del nódulo puncionado. Hubo 13 hombres $(9,8 \%)$ y 120 mujeres $(90,2 \%)$. El grupo de edad de mayor incidencia fue entre los 41 y los 60 años $(51,9 \%)$, seguido del grupo entre 21 y 40 años $(26,3 \%)$ (Tabla 1$)$.

Los nódulos puncionados cuya punción preoperatoria indicaba "neoplasia folicular", tras su estudio 
Tabla 1. Características del grupo estudiado

\begin{tabular}{|lrc|}
\hline Variable & n & $(\%)$ \\
\hline Grupos por edad (años) & & \\
$\leq 20$ & 6 & $(4,5)$ \\
$21-40$ & 35 & $(26,3)$ \\
$41-60$ & 69 & $(51,9)$ \\
$>60$ & 23 & $(17,3)$ \\
Total & $133(100)$ \\
Sexo & & \\
Masculino & 13 & $(9,8)$ \\
Femenino & 120 & $(90,2)$ \\
Total & $133(100)$ \\
\hline
\end{tabular}

Tabla 2. Histología definitiva del nódulo puncionado

\begin{tabular}{|lc|}
\hline $\begin{array}{l}\text { Histología definitiva del nódulo } \\
\text { puncionado }\end{array}$ & n (\%) \\
Hiperplasia folicular coloidea & $69(51,9)$ \\
\hline Adenoma folicular & $35(26,3)$ \\
\hline Carcinoma folicular & $9(6,8)$ \\
\hline Tiroiditis de Hashimoto & $6(4,5)$ \\
\hline Carcinoma papilar variedad folicular & $5(3,7)$ \\
Carcinoma papilar variedad usual & $4(3,0)$ \\
$\begin{array}{l}\text { Hiperplasia folicular multinodular con } \\
\text { metaplasia de células de Hürtle }\end{array}$ & $3(2,3)$ \\
\begin{tabular}{l} 
Carcinoma medular \\
\hline
\end{tabular} & $2(1,5)$ \\
\hline
\end{tabular}

Tabla 3. Histología definitiva de la glándula tiroides completa (se indica la principal histología de la muestra)

\begin{tabular}{|lc|}
\hline $\begin{array}{l}\text { Histología definitiva de tiroides total } \\
\text { (se indica la principal histología de la } \\
\text { muestra) }\end{array}$ & n (\%) \\
\hline $\begin{array}{l}\text { Hiperplasia folicular coloídea } \\
\text { Adenoma folicular }\end{array}$ & $58(43,6)$ \\
\hline Carcinoma papilar variedad usual & $29(21,8)$ \\
\hline Carcinoma papilar variedad folicular & $19(14,3)$ \\
\hline $\begin{array}{l}\text { Carcinoma folicular } \\
\text { Tiroiditis de Hashimoto }\end{array}$ & $9(6,8)$ \\
\hline $\begin{array}{l}\text { Hiperplasia folicular multinodular con } \\
\text { Metaplasia de células de Hürthle }\end{array}$ & $5(6,8)$ \\
\hline Carcinoma medular & $2(1,5)$ \\
\hline
\end{tabular}

anatomopatológico definitivo, correspondieron en su mayoría a hiperplasia folicular (51,9\%), seguido de adenoma folicular (26,3\%) y carcinoma folicular $(6,8 \%)$. La prevalencia de malignidad de cualquier histología en el nódulo puncionado fue finalmente de $15 \%$ (Tabla 2).

La histología definitiva de la muestra completa (incluyendo los hallazgos del nódulo puncionado) correspondieron en su mayoría a hiperplasia folicular (43,6\%), seguido de adenoma folicular $(21,8 \%)$ y carcinoma papilar (variedad usual $14,3 \%$, variedad folicular $6,8 \%$ ). No existía concordancia entre la histología definitiva del nódulo puncionado y la de la muestra de tiroides total (Kappa 0,598) debido principalmente al hallazgo de lesiones malignas incidentales en el resto de la tiroides (Tabla 3).

La prevalencia de malignidad en la muestra de tiroides total fue de $29 \%$, significativamente mayor que la del nódulo puncionado, de sólo $15 \%\left(\chi^{2} 56,7\right.$, $\mathrm{p}<0,001$ ) (Figura 1).

Los hallazgos malignos incidentales en otra zona distinta al nódulo puncionado ocurrieron en 19 pacientes $(14,2 \%)$ y correspondieron en un $100 \%$ a carcinoma papilar (15 carcinomas papilares de variedad usual y 4 carcinomas papilares de variedad folicular). La histología definitiva del nódulo pun-

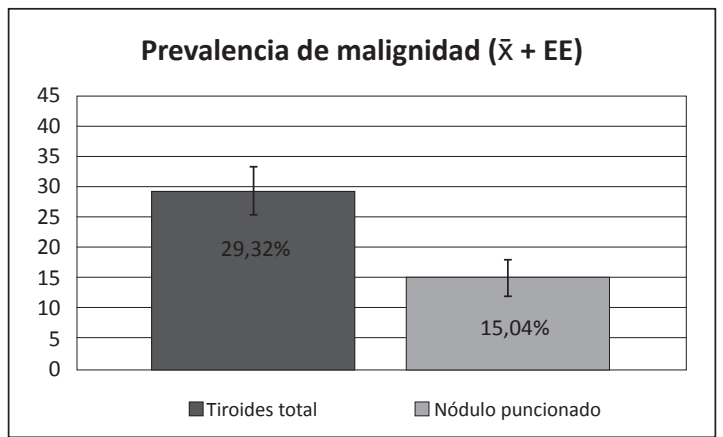

Figura 1. Prevalencia de malignidad.

Tabla 4. Histología definitiva del nódulo puncionado en los pacientes con hallazgos malignos adicionales

\begin{tabular}{|lr|}
\hline $\begin{array}{l}\text { Histología definitiva del nódulo } \\
\text { puncionado en los pacientes con } \\
\text { hallazgos malignos adicionales. }\end{array}$ & n (\%) \\
\hline Hiperplasia folicular coloidea & $11(57,9)$ \\
\hline $\begin{array}{l}\text { Adenoma folicular } \\
\text { Tiroiditis de Hashimoto }\end{array}$ & $1(31,6)$ \\
\hline $\begin{array}{l}\text { Hiperplasia folicular multinodular con } \\
\text { metaplasia de células de Hürthle }\end{array}$ & $1 \quad(5,3)$ \\
\hline
\end{tabular}


HALLAZGOS ANATOMOPATOLÓGICOS DEFINITIVOS EN PACIENTES TIROIDECTOMIZADOS CON DIAGNÓSTICO...

cionado en los pacientes en los cuales hubo hallazgo de lesiones malignas en la muestra total fueron principalmente hiperplasia folicular $(57,9 \%)$ y adenoma folicular (31,6\%) (Tabla 4).

\section{Discusión}

La PAAF para el estudio de nódulos tiroideos está ampliamente aceptada por su adecuada relación costo-eficiencia, seguridad y precisión diagnóstica ${ }^{1-5}$. Sin embargo, existe entre un 10 y $20 \%$ de resultados indeterminados, la mayoría de ellos por neoplasias foliculares (en alrededor del 65\% de los $\operatorname{casos}^{1,2}$ ).

Los pacientes estudiados no difieren demográficamente de lo publicado en la literatura ${ }^{2,9}$. Los nódulos de la glándula tiroides son más frecuentes en mujeres y en los rangos de edad señalados en la Tabla $1^{9}$.

Una PAAF que informa un diagnóstico "indeterminado", obliga a la cirugía total o parcial de la tiroides. En los pacientes estudiados se realizó siempre una tiroidectomía total, aunque no hubiese nódulos contralaterales, por estandarización del procedimiento, y con el debido consentimiento informado. Por otro lado, como se evidenció en los resultados, los hallazgos de patología maligna son frecuentes, lo que apoya la realización de una tiroidectomía total inicial. No se utilizó biopsia intraoperatoria, siguiendo las recomendaciones vigentes ${ }^{9}$. Aunque el principal propósito de la obtención de la pieza postoperatoria completa es diferenciar entre una neoplasia folicular maligna o benigna, con frecuencia el resultado final informa histologías no foliculares, tanto benignas como malignas en el nódulo puncionado y/o en el resto de la glándula.

El diagnóstico histológico definitivo en el nódulo puncionado fue de $33,1 \%$ de neoplasias foliculares verdaderas y de $15 \%$ de malignidad (cáncer folicular, papilar o medular). Además, en el $29 \%$ de los casos hubo finalmente cáncer de algún tipo en la glándula completa. Estos dos últimos hechos, apoyan la indicación de cirugía. Estas cifras son similares a la de otras series. La interpretación citológica e histológica de las neoplasias foliculares por PAAF es compleja y de difícil diagnóstico diferencial ${ }^{4,10}$. De esta manera, es esperable en el diagnóstico definitivo lesiones tumorales diversas.

En nuestro centro no realizamos estudios de la muestra obtenida por PAAF con marcadores inmunohistoquímicos o genéticos, ya que su utilización no está estandarizada, no agrega determinaciones terapéuticas distintas a la tiroidectomía y aumentan el costo. Su utilidad es motivo de investigaciones, especialmente en relación a la combinación necesaria de marcadores, ya sea de mayor sensibilidad y/o especificidad ${ }^{1}$. En casos seleccionados en que la indicación quirúrgica es discutible, podrían utilizarse estos métodos de diferenciación entre benignidad y malignidad, para definir una eventual cirugía.

A los hallazgos histopatológicos definitivos del nódulo puncionado (Tabla 2), se agregan con frecuencia otros diagnósticos del resto de la glándula. Los análisis mostrados en este estudio, señalan que la prevalencia de neoplasias malignas de cualquier tipo fue significativamente mayor en la muestra total que en el nódulo puncionado. En este grupo se enmarcan los hallazgos de microcarcinomas y carcinomas diferenciados incidentales ${ }^{11}$, mayoritariamente.

También se analizó el hallazgo de malignidad en el resto de la glándula tiroides. La prevalencia fue de 14,2\%, siendo sólo cánceres papilares. Esto está de acuerdo con hallazgos previamente publicados en que se muestra una alta prevalencia de carcinomas papilares incidentales en las tiroidectomías por otras causas $^{12}$

De este estudio se concluye que el informe de "neoplasia folicular" significa un riesgo alto de lesiones efectivamente tumorales foliculares o de otros tipos. Los hallazgos encontrados en el resto de glándula presentan también una prevalencia importante, lo que justificaría la indicación quirúrgica de tiroidectomía total.

\section{Referencias}

1. Meredith K, Thomas F. Molecular Markers in Thyroid Cancer Diagnostics. Surg Clin N Am. 2009;89:113955 .

2. Lew J, Snyder R, Sánchez Y, Solorzano C. Fine Needle Aspiration of the Thyroid: Correlation with Final Histopathology in a Surgical Series of 797 Patientes. J Am Coll Surg. 2011;213:188-94.

3. Baloch ZW, LiVolsi VA, Asa SL, Rosai J, Merino MJ, Randolph G, et al. Diagnostic terminology and morphologic criteria for cytological diagnosis of thyroid lesions: a synopsis of the National Cancer Institute Thyroid Fine-Needle Aspiration State of the Science Conference. Diagn Cytopathol 2008;36:425-37.

4. Saleh H, Jin B, Barnwell J, Alzohaili O. Utility of inmunohistochemical markers in differentiating benign from malignant follicular derived thyroid nodules. Diagnostic Pathology 2010;5:9.

5. Finley DJ, Zhu B, Barden CB. Discrimination of benign and malignant thyroid nodules by molecular profiling. Ann Surg. 2004;240:425-36.

6. Roh M, Jo V, Stelow E, Faquin W, Zou K, Alexander E, et al. The Predictive Value of the Fine-Needle Aspiration Diagnosis "Suspicious for a Follicular Neoplasm, Hürthle Cell Type" in Patientes with Hashimoto Thyroiditis. Am J Clin Pathol. 2011;135:139-44.

7. Keskek M, Ocak S, Ozalp N, Tez M. Clinical Signi- 
I. GOÑI E. y cols.

ficance of Hürthle Cells in Fine Needle Aspiration Biopsy for Multinodular Goiter. Endocrine Regulations 2010;44:65-8.

8. Turanli S, Pirhan Y, Ozcelik C, Cetin A. Predictors of Malignancy in Patients with a Thyroid Nodule that contains Hürthle Cells. Otolaryngol Head Neck Surg. 2011;144:514-7.

9. Alonso N, Lucas A, Salinas I, Castella E, Sanmartí A. Frozen Section in a Citological Diagnosis of Thyroid Follicular Neoplasm. Laryngoscope 2003;113:563-6.

10. Oestreicher-Kedern Y, Halpen M, Roizman P, Hardy B,
Sulkes J, Feinmesser R, et al. Diagnostic value of galectina-3 as marker for malignancy in follicular patterned thyroid lesions. Head Neck 2004;26:1960-6.

11. Fardella C, Jiménez M, González H, León A, Goñi I, Cruz F, y cols. Características de presentación del microcarcinoma papilar del tiroides. Experiencia retrospectiva de los últimos 12 años. Rev Méd Chile 2005;133:1305-10.

12. Bradly DP, Reddy V, Prinz RA, Gattuso P. Incidental papillary carcinoma in patients treated surgically for benign thyroid diseases. Surgery 2009;146:1099-104. 